\title{
Analysis of axial response of submarine pipeline to debris flow loading
}

Indranil Guha ${ }^{1}$, Mark F. Randolph ${ }^{2}$ and David J. White ${ }^{3}$

Technical Note submitted to Journal of Geotechnical and Geoenvironmental Engineering

Manuscript GTENG-9929: Revised 26 ${ }^{\text {th }}$ June 2020

${ }^{1}$ Indranil GUHA

Centre for Offshore Foundation Systems, University of Western Australia

35 Stirling Highway

Perth, WA 6009

Australia

Tel: +61 425531980

Email: guhanil@gmail.com

${ }^{2}$ Mark F. RANDOLPH (corresponding author)

Centre for Offshore Foundation Systems, University of Western Australia

35 Stirling Highway

Perth, WA 6009

Australia

Tel: +61 421586075

Email: mark.randolph@uwa.edu.au

${ }^{3}$ David J White

Faculty of Engineering and Physical Sciences

University of Southampton

University Road

Southampton, SO17 1BJ

UK

Tel: +4402380 596859

Email: david.white@soton.ac.uk

No. of words: 1975 (without abstract and references)

No. of tables: 2

No. of figures: 4 


\section{Analysis of axial response of submarine pipeline to debris flow loading}

\section{ABSTRACT}

4 This technical note presents simplified parametric solutions for the axial response of surface-

5 laid submarine pipelines subjected to axial drag from debris flows. In assessing the response of

6 pipelines impacted by debris flow emanating from a submarine landslide, both normal and axial

7 responses must be considered. Previous work has indicated that these can be decoupled, at least

8 as a first stage analysis. The most critical aspect of axial drag is the potential for the pipeline to

9 buckle. However, in order to make preliminary estimates of displacements and forces along the

10 pipeline prior to buckling, simple assumptions of elastic pipeline response with elastic perfectly

11 plastic interaction with the seabed are justified. These allow the development of parametric

12 solutions that contain only three non-dimensional quantities. The technical note documents the

13 solutions and illustrates their application for some typical input conditions.

\section{KEYWORDS}

15 Analysis, axial response, debris flow, submarine pipelines 
17

\section{INTRODUCTION}

The offshore oil and gas industry commonly operates in deep water, beyond the continental shelf, where infrastructure is vulnerable to a number of geohazards including submarine landslides, mud and volcanoes, seismicity, shallow gas and gas hydrates (Kvalstad et al., 2001). One of the most significant geohazards on the continental slope is the threat of submarine landslides, which typically originate from the shelf-break but may run out several kilometres into development zones or across pipeline routes. It is therefore necessary to consider both normal and axial responses of pipelines impacted by debris flow, although the two modes of response can be decoupled, at least as a first stage analysis (Randolph et al., 2010). Attention here is focused on the axial response.

The most critical aspect of axial drag is the potential for the pipeline to buckle due to compressive loading. However, in order to make preliminary estimates of displacements and forces along the pipeline prior to buckling, it is sufficient to consider purely elastic response of the pipeline, together with elastic perfectly plastic interaction with the seabed. The axial drag resulting from the submarine slide may be considered as a uniform traction applied to the pipeline over a defined zone.

These simple assumptions allow the development of parametric solutions to the problem that contain only three non-dimensional quantities. The technical note documents the solutions and illustrates their application for some typical input conditions. As an aside, it may also be noted that many of the underlying relationships presented here may also be applied to related pipeline problems, such as thermal expansion and contraction.

\section{PROBLEM DEFINITION}

The submarine slide-pipeline-seabed interaction problem may be divided into three parts: active slide zone, passive plastic zone and elastic zone as shown in Figure 1. Within the slide zone, 
the axial drag, $F_{\text {slide, }}$, is assumed to overcome the 'passive' seabed resistance, resulting in a net traction of $F_{\text {net }}$ applied over the width of the submarine impact zone. Nominally this may be considered as the difference between the slide loading and the passive seabed resistance, although in practice the latter may be modified, and even eliminated, within the slide zone. Beyond that zone, the seabed provides either an axial load-transfer stiffness (in the far-field 'elastic' zone) or a limiting passive resistance $F_{\text {passive }}$ within the intermediate 'passive' zone. Key axial tractions within each zone, and the loads and displacements at the interface points between zones, are indicated in the schematic. The response in each zone is solved analytically for the relevant boundary conditions in the following sections.

\section{Input parameters and dimensionless groups}

The perfectly straight pipe is defined by diameter, $D$, wall thickness, $t$ submerged unit weight, $W^{\prime}$, and Young's modulus, $E$, from which the axial rigidity $E A$ can be calculated. The slide is defined as a block zone of length, $L_{\text {slide }}$; from symmetry of the problem, only the half slide length, $L_{\mathrm{AB}}$ is considered here, with the axial force $P_{\mathrm{A}}$ in the pipeline at the centre of the slide zone taken as zero. Any existing axial force distribution in the pipeline is ignored here, although it would be relatively straightforward to extend the solutions presented to allow for that. The length of the 'passive plastic zone' is $L_{\mathrm{BC}}$, beyond which point ( $C$ onwards) the pipeline-seabed interaction is elastic (Figure 1). The displacement at the centre of the slide, $A$, is $u_{\mathrm{A}}$, at the interface of 'active' and 'passive' zones, $B$, is $\mathrm{u}_{\mathrm{B}}$ and at the interface between 'passive' and 'elastic' zones, $C$ is $u_{\mathrm{c}}$. The axial load generated within the pipeline due to the slide movement along the length is defined as $P$. The loads take values of $P_{\mathrm{A}}, P_{B}$ and $P_{\mathrm{C}}$ at the points corresponding with $u_{\mathrm{A}}, u_{\mathrm{B}}$ and $u_{\mathrm{C}}$.

The axial load transfer stiffness between pipeline and seabed has been considered by Guha et al. (2016). For a partially embedded pipeline contacting the seabed over chord width $D^{\prime}$ (i.e. with $0<D^{\prime} \leq D$ ), the elastic load transfer stiffness may be approximated as 


$$
F / u=k_{x}=G_{D^{\prime}}
$$

66

where $G_{\mathrm{D}^{\prime}}$ is the seabed shear modulus at a depth of the pipeline-seabed contact width $D^{\prime}$. More detailed expressions, allowing for the pipeline embedment and exact profile of the seabed shear modulus, are provided by Guha et al. (2016) and extend over a range of $\pm 20 \%$ relative to the above approximation. However, given the inevitable uncertainty in estimating shear modulus values at very shallow depth, Equation (1) is considered sufficient.

The output quantities may be non-dimensionalised and expressed in terms of various input properties. The maximum axial load, $P_{B}$, may be normalised by the axial elastic stiffness of the pipe, $E A$, and presented as compressive strain, $\varepsilon=P / E A$; the axial displacement, $u$, may be normalised by the slide length, $L_{\text {slide }}$, as $u / L_{\text {slide }}$. These normalised output parameters may then be expressed in terms of normalised input parameters, i.e. the driving force, $a_{1}=F_{\text {net }} L_{\text {slide }} / E A$; passive resistance, $a_{2}=F_{\text {passive }} L_{\text {slide }} / E A$; and pipe-soil stiffness, $a_{3}=k_{\mathrm{x}} L_{\text {slide }}{ }^{2} / E A$. These three groups can be shown to be sufficient to determine the longitudinal profile of load and displacement of the pipe non-dimensionally. 
80 Table 1 summarises the problem variables, together with relevant ranges for each that are

81 considered later. The range for the pile-soil axial stiffness $k_{x}$ is quite large, reflecting conditions

82 from a small $(0.1 \mathrm{~m})$ diameter pipe half embedded in a soft clay with shear modulus of perhaps

$83500 \mathrm{kPa}$, to a large $(1 \mathrm{~m})$ diameter pipe shallowly embedded in dense sand with $G_{D^{\prime}} \sim 10 \mathrm{MPa}$.

84 From a practical point of view, very high combinations of the net force and length of slide 85 impact will lead to buckling of the pipeline, which is outside the scope of the solutions presented 86 here (see Guha, 2020), or at least localised plastic yield. Since the maximum normalised force 87 induced in the pipeline (i.e. average axial strain in the pipe) is, by inspection, $a_{1} / 2$, an upper 88 limit of the normalised slide force is about 0.004 for elastic conditions to be maintained, and 89 rather less than that once buckling is considered. 
ANALYTICAL SOLUTION

\section{Elastic zone}

93 The axial load generated in the pipe due to the presence of frictional resistance of the seabed is

$$
\frac{d P}{d x}=-F
$$

94 The compressive strain, $\varepsilon_{\mathrm{x}}$, of the pipeline (assumed elastic) is written in terms of the load, $P$,

95 transmitted by the pipe at any length $x$,

$$
\varepsilon=-\frac{d u}{d x}=\frac{-P}{E A}
$$

96 Differentiating equation (3) and using equations (1) and (2) gives

$$
\frac{d^{2} u}{d x^{2}}=\frac{F}{E A}=\frac{k_{x}}{E A} u
$$

97 The solution of this equation is

$$
u(x)=C_{2} e^{\lambda x}+C_{2} e^{-\lambda x}
$$

98 where $\lambda=\sqrt{k_{x} / E A}$ is the inverse of a characteristic length with dimensions $\mathrm{m}^{-1}$. To satisfy

99 the boundary conditions of zero displacement at large $x$, and $u=u_{C}$ at $x=x_{C}$, the displacement

100 variation within the elastic zone be expressed in terms of the displacement at the passive-plastic

101 and elastic zone interface by:

$$
u(x)=u_{C} e^{-\lambda\left(x-x_{C}\right)}
$$

102 The profile of load in the pipe may then be obtained by substituting equation (6) into equation

103 (3) and integrating to yield: 


$$
P=\frac{k_{x}}{\lambda} u_{C} e^{-\lambda\left(x-x_{C}\right)}
$$

104 from which

$$
P_{C}=\sqrt{k_{x} E A} u_{C}
$$

105 In non-dimensional form, this may be written as

$$
\frac{P_{C}}{E A}=\sqrt{\frac{k_{x} L_{\text {slide }}^{2}}{E A}} \frac{u_{C}}{L_{\text {slide }}}=\sqrt{a_{3}} \frac{u_{C}}{L_{\text {slide }}}
$$

106 In principle, point $C$ represents the interface between passive plastic and elastic zones (see

107 Figure 1), although if the active slide force is small the passive plastic zone may disappear. An

108 upper limit for the displacement at $C$ is

$$
u_{C-\text { slip }}=\frac{F_{\text {passive }}}{k_{x}} \text { hence } \frac{u_{C-\text { slip }}}{L_{\text {slide }}}=\frac{F_{\text {passive }} L_{\text {slide }}}{E A} \frac{E A}{k_{x} L_{\text {slide }}^{2}}=\frac{a_{2}}{a_{3}}
$$

109 Substituting this into equation (9) gives the maximum load at the boundary of the elastic zone,

110 for the long pipe considered here, as

$$
P_{C, \max }=F_{\text {passive }} \sqrt{\frac{E A}{k_{x}}} \text { hence } \frac{P_{C, \max }}{E A}=\frac{a_{2}}{\sqrt{a_{3}}}
$$

\section{Passive plastic zone}

112 In general, there will be a passive plastic zone between the active slide zone and the elastic

113 zone, where slip occurs between the seabed and the pipe and the resistance force per unit length

114 is $F_{\text {passive. }}$ The governing equations of the plastic zone are similar to those for the elastic zone,

115 but with $F=F_{\text {passive }}$ in equation (2). This results in a linear increase in force in the pipe between

116 points $\mathrm{C}$ and $\mathrm{B}$, with 


$$
P_{B}=P_{C}+F_{\text {passive }} L_{B C} \text { hence } \frac{P_{B}}{E A}=\frac{P_{C}}{E A}+a_{2} \frac{L_{B C}}{L_{\text {slide }}}
$$

117 This may be used to determine the length of the passive zone, $L_{\mathrm{BC}}$ as

$$
\frac{L_{B C}}{L_{\text {slide }}}=\frac{1}{a_{2}}\left(\frac{P_{B}}{E A}-\frac{P_{C}}{E A}\right)
$$

118 When the passive zone $L_{\mathrm{BC}}=0$, point $B$ coincides with point $C$ leading to $P_{\mathrm{B}}=P_{\mathrm{C}}$. In general,

119 though, we may write $P_{B} \geq P_{C}$ and $L_{B C} \geq 0$.

120 Integration of equation (3), allowing for the linear variation of $P$ between $\mathrm{B}$ and $\mathrm{C}$, yields

$$
\frac{u_{B}}{L_{\text {slide }}}-\frac{u_{C}}{L_{\text {slide }}}=\frac{\left(P_{B}+P_{C}\right)}{2 E A} \frac{L_{B C}}{L_{\text {slide }}}=\frac{1}{2}\left[\left(\frac{P_{B}}{E A}\right)^{2}-\left(\frac{P_{C}}{E A}\right)^{2}\right] \frac{E A}{F_{\text {passive }} L_{\text {slide }}}=\frac{1}{2 a_{2}}\left[\left(\frac{P_{B}}{E A}\right)^{2}-\left(\frac{P_{C}}{E A}\right)^{2}\right]
$$

121 For a small active slide load (or strong passive resistance), $u_{C}$ may not reach the elastic limit of $u_{C-\text { slip }}$, in which case $L_{B C}=0, P_{B}=P_{C}$ and $u_{B}=u_{C}$.

\section{Active zone}

124 In the active zone the interaction between the pipe and the soil is assumed to be plastic. The

125 displacement is taken as $u_{A}$ at the centre of the slide $(x=0)$ from symmetry. Similarly, the axial

126 force $P_{A}$ in the pipe is zero at $x=0$, and increases linearly to $P_{B}$ at the edge of the slide material,

127 where

$$
P_{B}=\frac{F_{\text {net }} L_{\text {slide }}}{2} \text { hence } \frac{P_{B}}{E A}=\frac{F_{\text {net }} L_{\text {slide }}}{2 E A}=\frac{a_{2}}{2}
$$

128 Note that $P_{B}$ represents the largest axial force generated in the pipeline, and hence the maximum

129 compressive strain in the pipe is $\varepsilon_{\max }=a_{1} / 2$.

130 Integrating equation (3), for the linear variation of $P$ from zero at $\mathrm{A}$ to $a_{1} / 2$ at $\mathrm{B}$, yields 


$$
u_{A}-u_{B}=\frac{P_{B} L_{\text {slide }}}{4 E A}=\frac{F_{\text {net }} L_{\text {slide }}^{2}}{8 E A} \text { hence } \frac{u_{A}}{L_{\text {slide }}}=\frac{u_{B}}{L_{\text {slide }}}+\frac{a_{1}}{8}
$$

\section{Summary of solution}

132 For convenience the main expressions are summarized here in non-dimensional form. The key

133 loads may be expressed as

$$
\frac{P_{A}}{E A}=0 ; \frac{P_{B}}{E A}=\frac{a_{1}}{2} ; \frac{P_{C}}{E A}=\operatorname{Min}\left(\frac{a_{1}}{2}, \frac{a_{2}}{\sqrt{a_{3}}}\right)
$$

134 The length of the (plastic) passive zone is given by

$$
\frac{L_{B C}}{L_{\text {slide }}}=\operatorname{Max}\left(0, \frac{a_{1}}{2 a_{2}}-\frac{1}{\sqrt{a_{3}}}\right)
$$

135 The displacements at key points are

$$
\begin{aligned}
\frac{u_{A}}{L_{\text {slide }}} & =\frac{a_{1}}{8}+\operatorname{Max}\left(\frac{a_{1}}{2 \sqrt{a_{3}}}, \frac{1}{8} \frac{a_{1}^{2}}{a_{2}}+\frac{1}{2} \frac{a_{2}}{a_{3}}\right) \\
\frac{u_{B}}{L_{\text {slide }}} & =\operatorname{Max}\left(\frac{a_{1}}{2 \sqrt{a_{3}}}, \frac{1}{8} \frac{a_{1}^{2}}{a_{2}}+\frac{1}{2} \frac{a_{2}}{a_{3}}\right) \\
\frac{u_{C}}{L_{\text {slide }}} & =\operatorname{Min}\left(\frac{1}{2} \frac{a_{1}}{\sqrt{a_{3}}}, \frac{a_{2}}{a_{3}}\right)
\end{aligned}
$$

136 These relationships are illustrated in Figure 2, which shows the length of the plastic zone $L_{B C}$

137 as a function of the normalised net slide force $a_{1}=F_{\text {net }} L_{\text {slide }} /$ EA, and Figure 3, which shows

138 corresponding key displacement ratios. As might be expected intuitively, the length of the

139 plastic zone grows proportionally with the ratio of driving to resisting force $\left(F_{\text {net }} / F_{\text {passive }}\right)$, with

140 almost no influence of the elastic stiffness ratio $a_{3}=k_{x} L_{\text {slide }}{ }^{2} / E A$ apart from at very low ratios

141 of $F_{\text {net }} / F_{\text {passive }}$. 
142 In a similar vein, the magnitude of displacements $u_{A}$ and $u_{B}$, both normalised by $L_{\text {slide, }}$ grow

143 proportionally with the ratio of driving to resisting force, except where that ratio falls below

144 unity. Once $F_{n e t} / F_{\text {passive }}$ reduces below unity, the maximum displacement at the mid-point of the

145 slide $\left(u_{A}\right)$ asymptotes to a plateau that corresponds to the pipe compression within the slide

146 zone, essentially half the ratio $0.5 a_{2} / a_{3}$, as the displacement at $B$ reduces towards zero. In most

147 cases the displacement at interface between passive and elastic zones $\left(u_{C}\right)$ is negligible.

\section{EXAMPLE NUMERICAL SOLUTION}

149 As a check on the analytical solution, and to explore the effect of different slide loading on a

150 given pipeline, three example cases are considered here, with results compared with those

151 obtained from finite element analysis (Guha, 2020). The three cases were for a $1 \mathrm{~m}$ diameter

152 pipeline with $D / t$ of 25 , subjected to slide loading of $11.9 \mathrm{kN} / \mathrm{m}$ over slide lengths of 100,300

153 and $500 \mathrm{~m}$, beyond the seabed passive resistance is $3.8 \mathrm{kN} / \mathrm{m}$. The input data and corresponding

154 normalised parameters are summarised in Table 2.

155 Figure 4 shows the resulting profiles of (a) axial force, and (b) axial displacement along the

156 pipeline for the three cases. Note the axial displacements have been factored up by 1000 .

157 Corresponding displacements from finite element analyses (Guha, 2020) are shown for

158 comparison. The zones of slide loading, plastic passive resistance and elastic resistance are

159 colour coded, respectively blue, red and green. The finite element data confirm the accuracy of

160 the analytical solution. Overall, the results also show that, provided the pipe does not fail

161 through plasticity or buckling, the axial displacements remain rather small, varying

162 quadratically with the magnitude of total slide load $\left(F_{n e t} L_{\text {slide }}\right)$ and, for these cases, ranging

163 between $2.7 \mathrm{~mm}$ and $62 \mathrm{~mm}$. 
CONCLUDING REMARKS

165

166

This technical note has documented a simple analytical solution to the distribution of axial force, strain and displacements in a pipeline loaded axially by a debris flow. The solutions facilitate simple calculation of the potential for failure of a pipe due to plastic strains or (in a broader context not considered here) by lateral buckling.

\section{DATA AVAILABILITY STATEMENT}

All data, models, and code generated or used during the study appear in the submitted article.

\section{ACKNOWLEDGEMENTS}

This work forms part of the activities of the Centre for Offshore Foundation Systems (COFS), established in 1997 under the Australian Research Council's Special Research Centres Program, and supported (2010-2017) as a node of the Australian Research Council's Centre of Excellence for Geotechnical Science and Engineering, and through the Fugro Chair in Geotechnics, the Lloyd's Register Foundation Chair and Centre of Excellence in Offshore Foundations and the Shell Chair in Offshore Engineering.

\section{REFERENCES}

Guha, I. (2020). Structural Analysis of Submarine Pipelines Under Submarine Slide and Thermal Loading. Forthcoming PhD thesis, The University of Western Australia.

Guha, I., Randolph, M.F. and White, D.J. (2016). Evaluation of elastic stiffness parameters for pipeline-soil interaction. J. of Geotechnical and Geoenvironmental Engineering, ASCE, 04016009-(1-12).

Kvalstad, T.J., Nadim, F., and Harbitz, C.B. (2001). Deepwater geohazards: geotechnical concerns and solutions. Proc. Offshore Technology Conference, Houston, Paper OTC 12958. 
186 Randolph, M.F., Seo, D., and White, D.J. (2010). Parametric solution of slide impact on

187 pipelines. J. of Geotechnical and Geoenvironmental Engineering, 136(7), 940-949.

188 
189 Table 1: Summary of range of input and output parameters

\begin{tabular}{lll}
\hline Input parameters & Range & Units \\
\hline Pipeline diameter, $D$ & $0.1-1$ & $\mathrm{~m}$ \\
Pipeline diameter to wall thickness ratio, $D / t$ & $13-20$ & \\
Elastic modulus of pipeline, $E$ & 210 & $\mathrm{GPa}$ \\
Length of slide loading on pipeline, $L_{\text {slide }}$ & $50-1000$ & $\mathrm{~m}$ \\
Net slide force on pipeline, $F_{\text {net }}$ & $0.1-10$ & $\mathrm{kN} / \mathrm{m}$ \\
Passive seabed frictional resistance force, $F_{\text {passive }}$ & $0.02-10$ & $\mathrm{kN} / \mathrm{m}$ \\
Pipe-soil elastic axial stiffness, $k_{x}$ & $50-10,000$ & $\mathrm{kPa}$
\end{tabular}

Adopted range of non-dimensional input parameters

\begin{tabular}{lll}
\hline Normalised slide loading, $a_{1}$ & $\mathrm{~F}_{\text {net. }} \mathrm{L}_{\text {slide }} / \mathrm{EA}$ & $0.000001-0.01$ \\
Normalised passive resistance, $a_{2}$ & $\mathrm{~F}_{\text {passive. }} \mathrm{L}_{\text {slide }} / \mathrm{EA}$ & $0.000001-0.01$ \\
Normalised pipe-soil elastic stiffness, $a_{3}$ & $\mathrm{k}_{\mathrm{x} .} \mathrm{L}_{\text {slide }}$ (EA & $0.01-10000$
\end{tabular}

Non-dimensional output quantities

Axial loads $\quad \mathrm{P}_{\mathrm{B}} / \mathrm{EA}, \mathrm{P}_{\mathrm{C}} / \mathrm{EA}$

Length of passive zone $\quad \mathrm{L}_{\mathrm{BC}} / \mathrm{L}_{\text {slide }}$

Displacements $\quad \mathrm{u}_{\mathrm{A}} / \mathrm{L}_{\text {slide }}, \mathrm{u}_{\mathrm{B}} / \mathrm{L}_{\text {slide, }} \mathrm{u}_{\mathrm{C}} / \mathrm{L}_{\text {slide }}$ 
192 Table 2: Input parameters for numerical examples

\begin{tabular}{llll}
\hline Input parameters & Case 1 & Case 2 & Case 2 \\
\hline Pipeline diameter, $D(\mathrm{~m})$ & 1 & 1 & 1 \\
Diameter to wall thickness ratio, $D / t$ & 25 & 25 & 25 \\
Cross-sectional stiffness, $E A(\mathrm{MN})$ & 25300 & 25300 & 25300 \\
Length of slide, $L_{\text {slide }}(\mathrm{m})$ & 100 & 300 & 500 \\
Net slide loading, $F_{\text {net }}(\mathrm{kN} / \mathrm{m})$ & 11.9 & 11.9 & 11.9 \\
Passive resistance, $F_{\text {passive }}(\mathrm{kN} / \mathrm{m})$ & 3.8 & 3.8 & 3.8 \\
Pipe-soil elastic axial stiffness, $k_{x}(\mathrm{kPa})$ & 6400 & 7000 & 8000 \\
Normalised slide loading, $a_{1}$ & 0.000047 & 0.000141 & 0.000236 \\
Normalised passive resistance, $a_{2}$ & 0.000015 & 0.000045 & 0.000074 \\
Normalised pipe-soil elastic stiffness, $a_{3}$ & 2.5 & 24.9 & 78.9 \\
\hline
\end{tabular}

193

194

195

196

197 


\section{Figure Captions}

199 Figure 1 Idealisation of axial slide pipeline interaction

200 Figure 2 Length of passive zone, $L_{B C}$

201 Figure 3 Normalised axial displacements of pipe

202 Figure 4 Results of three example cases

203

204

205

206

207 


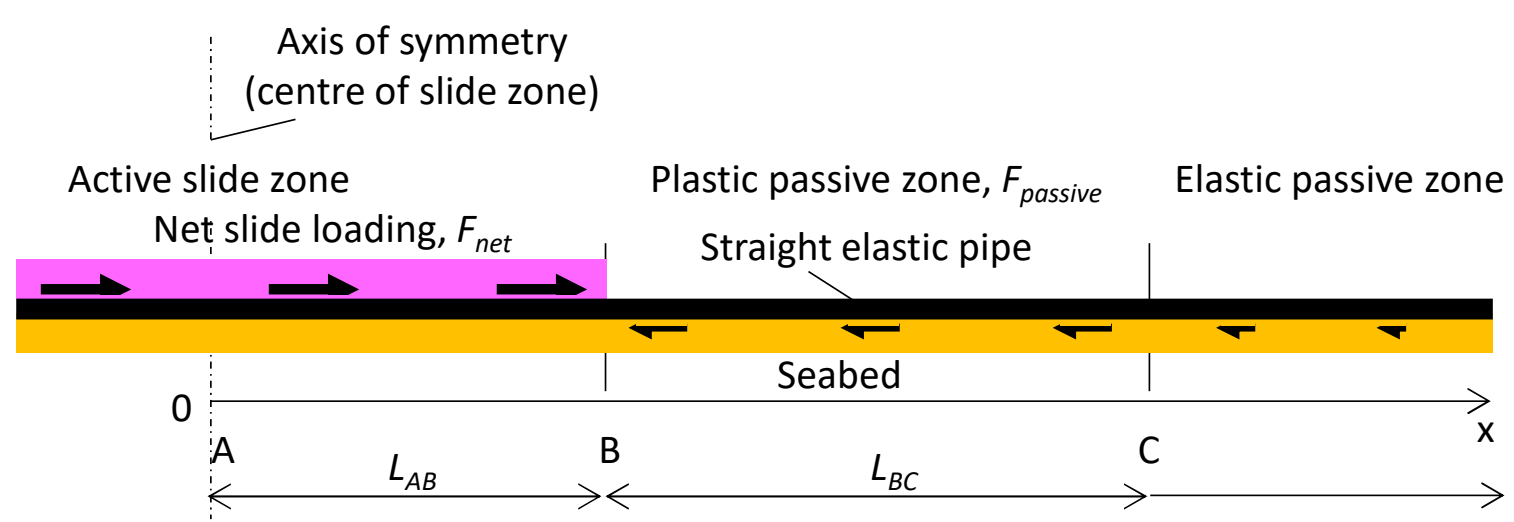

Figure 1 Idealisation of axial slide pipeline interaction 


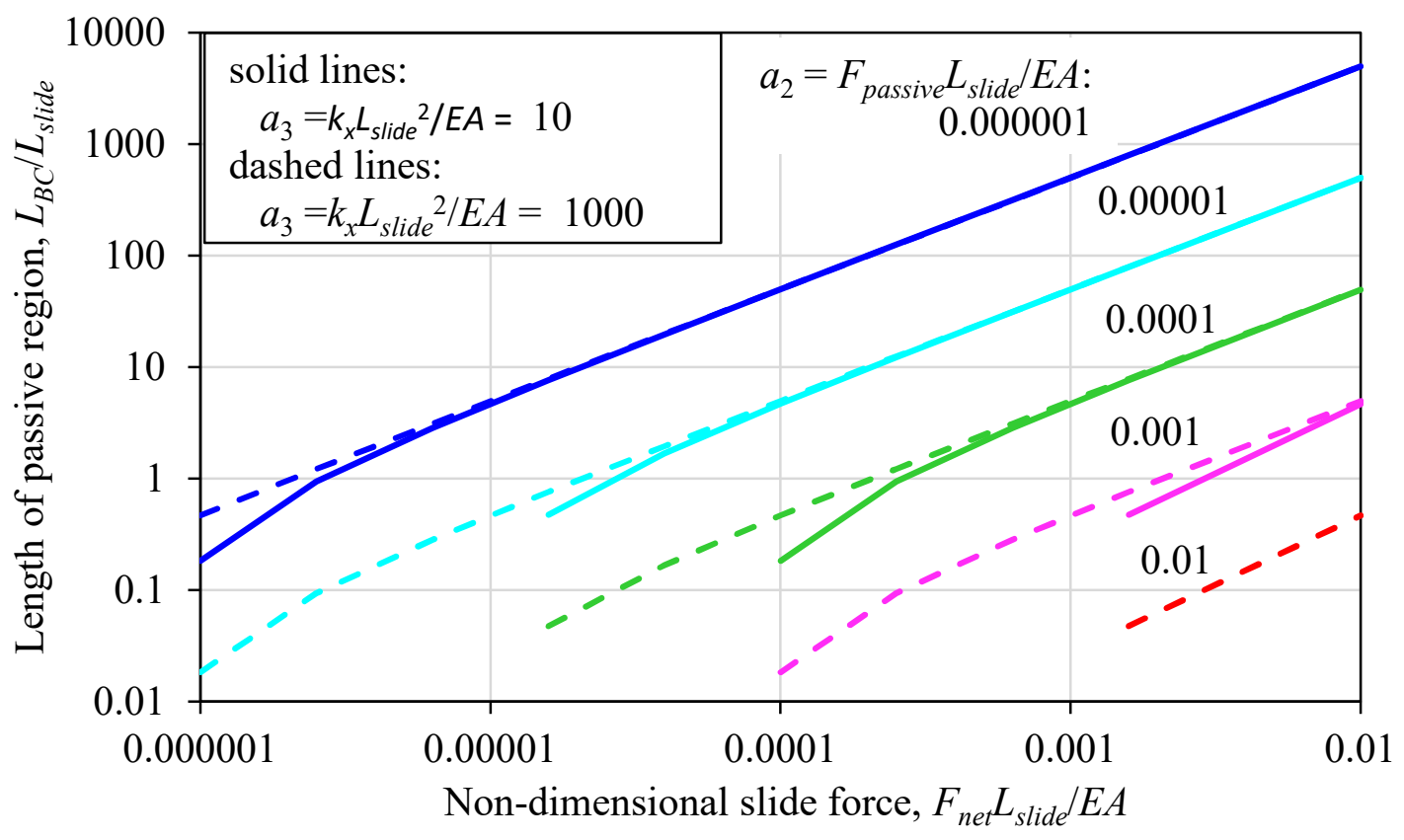

Figure 2 Length of passive zone, $L_{B C}$ 


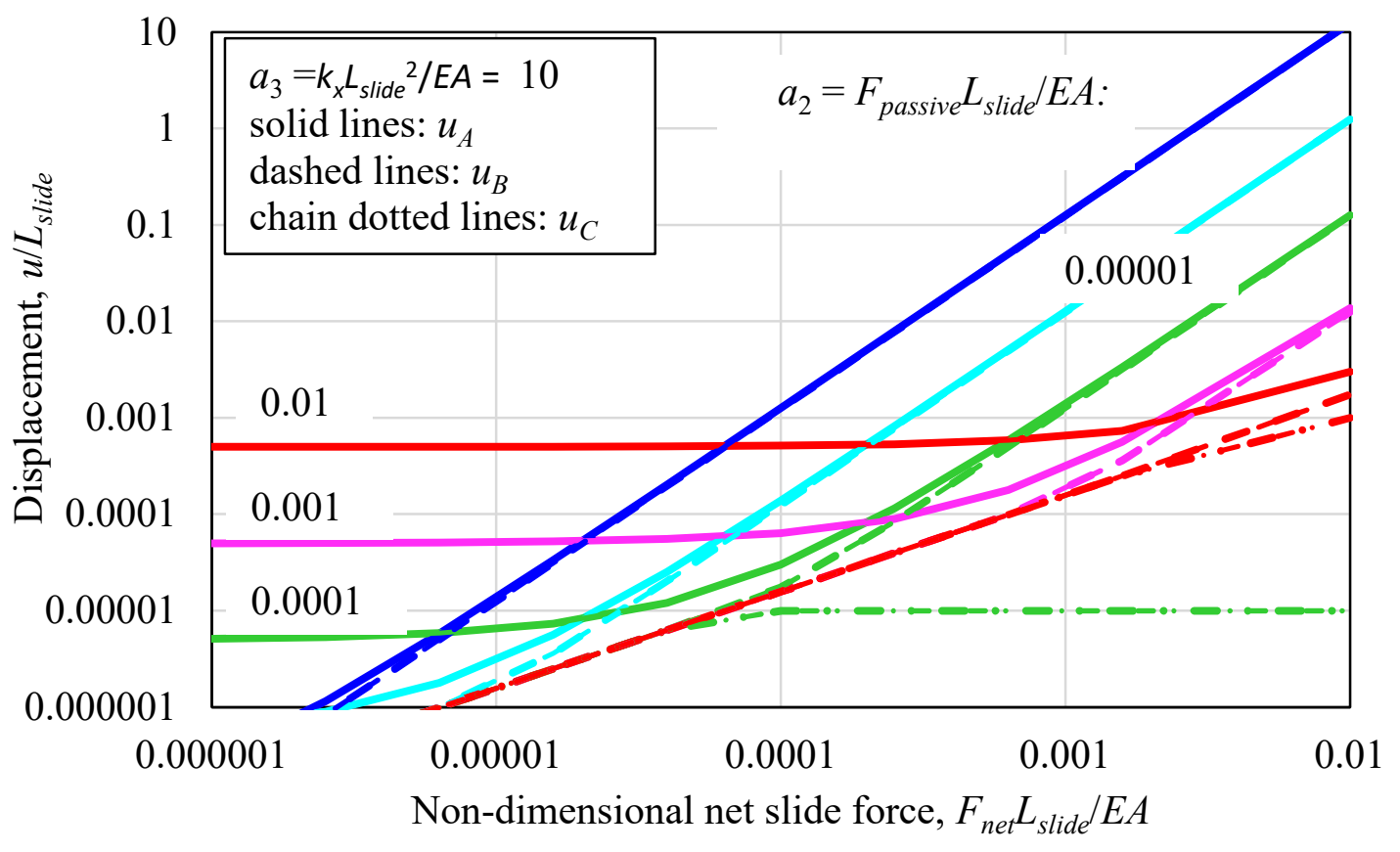

Figure 3 Normalised axial displacements of pipe 


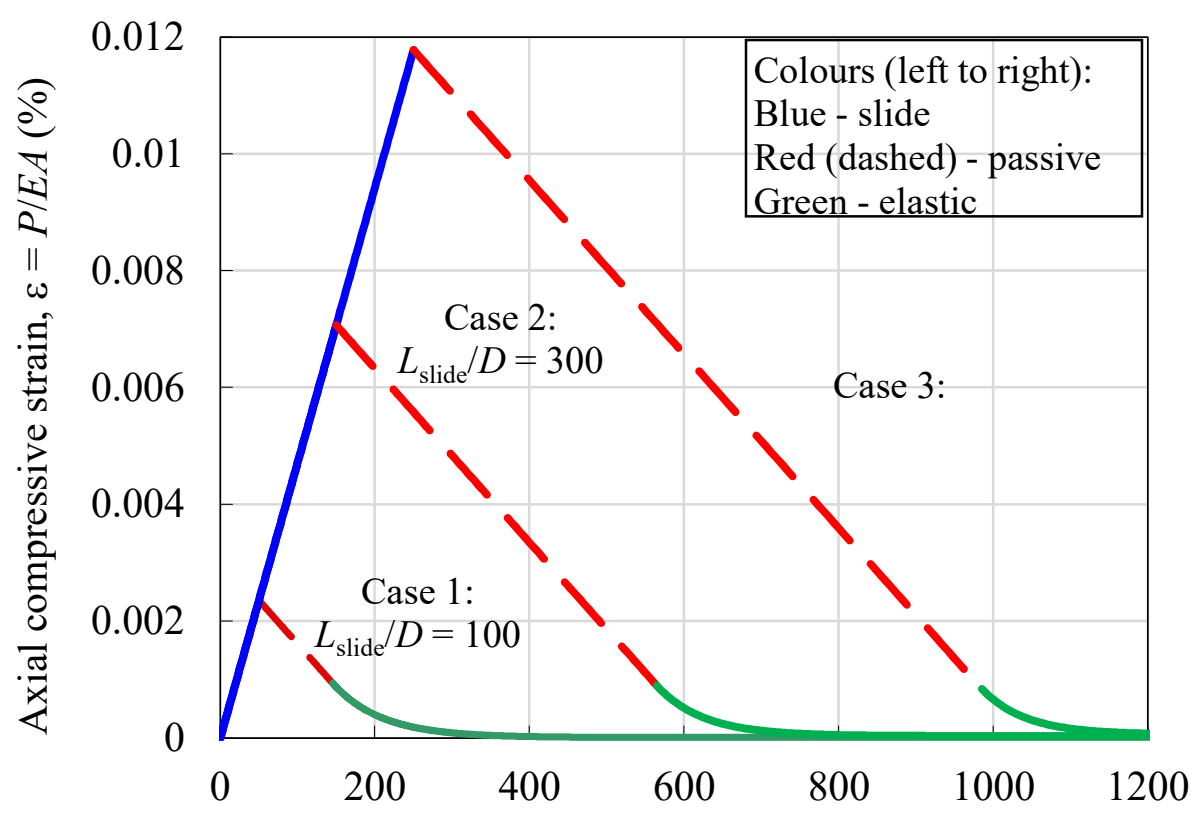

Distance along pipeline from slide centre, $x / D$

(a) Distribution of axial strain along the pipeline

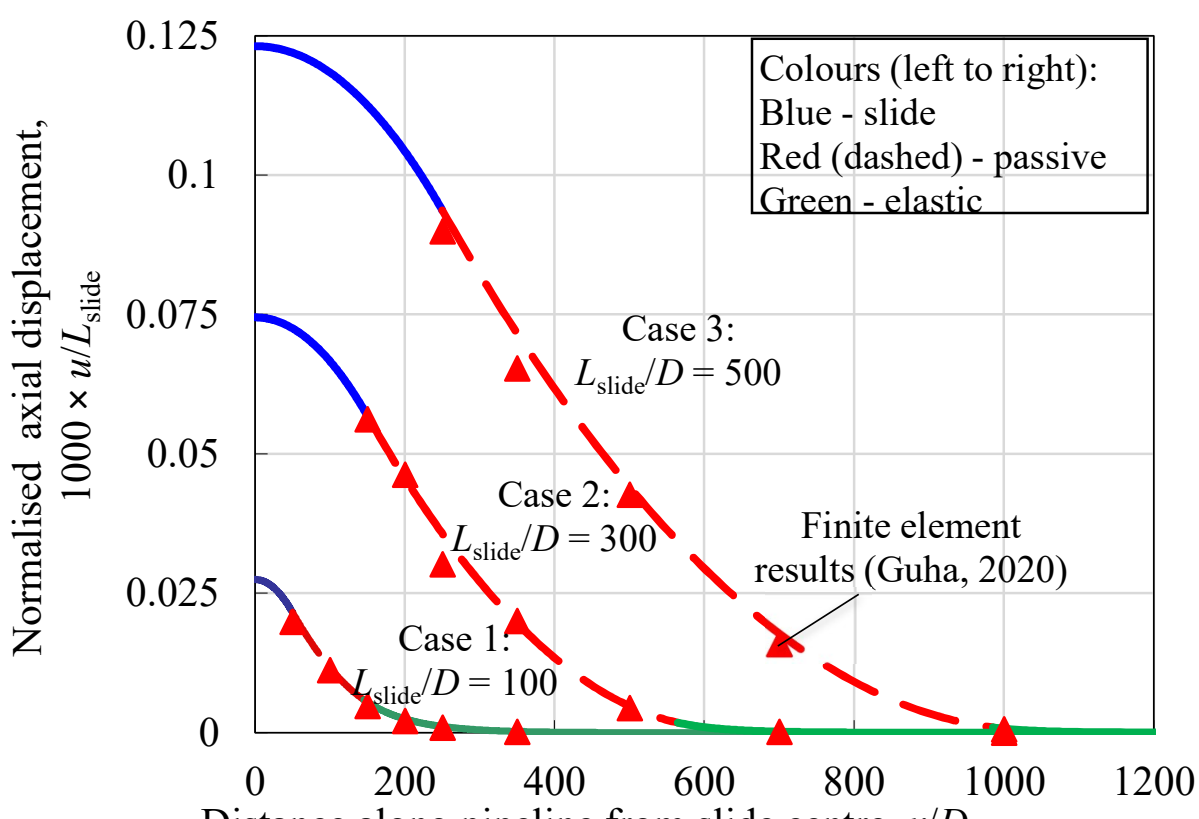

Distance along pipeline from slide centre, $x / D$

(b) Distribution of axial displacements along the pipeline

Figure 4 Results of three example cases 